\title{
Tradition and Tourism in Konavle
}

\section{Dr.Sc. Zrinka Režić Tolj}

\author{
Lecturer, University of Dubrovnik, Croatia
}

E-mail: zrinka.rezic@unidu.hr

\section{Doi:10.5901/ajis.2013.v2n3p209}

\begin{abstract}
The study presents a local example of traditional culture of Konavle (southernmost Croatian region) within the global tourist movement and it questions the possibility of applying ethnological and cultural-anthropological knowledge in the realm of tourism. Tourism in Konavle is a fitting example of the cultural-anthropological study of cultural tourism, especially in the realm of revival of tradition for the sake of tourism. This study gives a picture of the current situation and is a document representing the actual state of tradition in Konavle. It presents the use of folklore and particular cases of the revival of traditional manufacture (silk-worm breeding, flour milling) for the purpose of tourism. The study confirms that selected elements of traditional culture are significant factors of cultural and native identity of Konavle. It also shows that the cultural forms and the concepts of community identity are lasting and that they are transformed and influenced by contemporary tourist requirements and the interactions between different participants in tourism. The existing tourist offer shows Konavle as a tourist destination that accentuates the specific traditional culture of a micro-region with a strong local identity. Heritage, even though in a folklore packaging nowadays, forms a significant base for the development of this region. Through ensuring the livelihood for a considerable number of families in Konavle, tourism has a favourable influence on maintaining local traditions and identity.
\end{abstract}

Keywords: Konavle, culture, tradition, identity, tourism.

\section{Introduzione}

Il turismo contemporaneo è basato su evento ed esperienza particolari. La cultura, il patrimonio storico-culturale, le tradizioni e il folclore arricchiscono l'offerta turistica della destinazione offrendo ai turisti la pienezza della vicenda ed esperienze positive. II collegamento tra il turismo e l'etnologia / l'antropologia culturale è ovvio. Considerando che „analizzare il turismo corrisponde alla creazione della vera e propria etnologia della società moderna (Simonicca, 2001, p. 66), il turismo culturale ${ }^{1}$ è stato studiato in Konavle, una microregione di cui nei depliant turistici si potrebbe dire rappresenti una sorta di oasi della vita tradizionale. Nel mondo d'oggi la cultura tradizionale rappresenta (di solito) una curiosità molto attraente e nel turismo ha un significato speciale come „proprio quello che viene richiesto” perché al turista moderno presenta un mondo in cui c'è ancora un senso di connessione dell'individuo con la società e con la natura.

II lavoro rappresenta uno studio antropologico culturale della cultura tradizionale e locale di Konavle (la più meridionale regione croata), nel contesto del movimento turistico globale ed indaga la possibilità di applicare le conoscenze dell'antropologia culturale al mondo del turismo. Nell'interazione tra turismo e tradizione avviene spesso la sua interpretazione. Affinché la tradizione sia più autentica e fedele a sé stessa, essa deve soddisfare ugualmente le esigenze della popolazione locale (perché si tratta dei suoi vettori che devono credere veramente nella presentazione di sé stessi) e dei turisti (da cui soddisfazione semplicemente dipende il futuro della „variante" interpretata, ma in parte anche l'esistenza dei presentatori stessi). Per avere alla fine tutti soddisfatti (o usando il vocabolario turistico „per una strategia turistica di successo") è necessaria la cooperazione tra i titolari del patrimonio culturale locale e quelli del turismo, ma anche con gli esperti - etnologi / antropologi culturali.

Partendo dalla consapevolezza che il turismo è fatto da persone e sostenendo che in realtà, il turismo sono le persone, sono stati studiati i partecipanti (secondo il loro ruolo) al turismo, osservato alla luce della teoria (dell'interpretazione) della (vita come) rappresentazione sociale di Goffman (2000). In questo senso, il turismo è la rappresentazione sociale che tre parti (ognuna per sé e tutte insieme) creano per i turisti. Questo spettacolo viene diretto da operatori turistici che creano gite, programmi, eventi, „spettacoli” per i turisti e li introducono alla destinazione e alla sua gente, al loro modo di vita e alla loro cultura. In pratica, cioè sul campo si tratta del gruppo „invisibile” e meno presente dei partecipanti al turismo, che occupa tuttavia un posto e un ruolo molto importante nella creazione delle

\footnotetext{
${ }^{1}$ Si tratta del tipo ibrido del turismo con gli elementi dei vari aspetti del turismo selettivo.
} 
esperienze per i turisti, che sul campo creano le guide e la gente del posto. Gli operatori turistici mettono in contatto turisti, guide e residenti e permettono l'interazione tra di loro. Per quanto riguarda la rappresentazione stessa gli operatori turistici, anche se la dirigono, in pratica, per lo più (e tuttavia) non la guardano. Le guide sono registi veri in campo, ma anche gli attori, ai quali si possono associare gli abitanti locali, che sono anche le guide "dietro le quinte”. II turismo quindi, come una forma speciale di interazione umana, porta a contatto le persone diverse, i titolari di diverse culture, che nell'incontro turistico si rappresentano, riconoscono, apprendono e adattano l'una all'altra e quindi cambiano.

La ricerca sulla rappresentazione e la percezione di Konavle nel turismo dal titolo „Come presentiamo Konavle ai turisti?" e „Come i turisti percepiscono Konavle?" è stata condotta nel $2010^{2}$ in Konavle e a Dubrovnik. La metodologia della ricerca anonima si è basata sul questionario come strumento primario della ricerca, insieme con la tipica "osservazione partecipante" etnologica. Sono stati utilizzati 4 tipi di questionari ${ }^{3}$, preparati per i 4 tipi principali di partecipanti al turismo. I questionari strutturalmente simili erano costituiti da due parti: una generale, relativa ai soggetti intervistati ${ }^{4}$, e la seconda parte che riguardava $i$ temi della ricerca nel senso più ampio che conteneva 20 domande in tutti i tipi di questionari, eccetto il questionario per la popolazione locale con 32 domande. I risultati dell'indagine si basano sulle risposte di 152 persone: 16 operatori turistici che (creano ed) organizzano escursioni in Konavle, 37 guide che conducono il tour in Konavle, 44 residenti locali che lavorano nel turismo o sono in contatto con i turisti e 55 turisti che hanno visitato Konavle nel corso della ricerca. La tradizione come il tema più frequentemente citato nella ricerca, ha confermato la sua eccezionale importanza per Konavle in generale, per l'identità e la cultura di Konavle, ma anche per il turismo della stessa regione.

\section{Su Konavle}

La regione di Konavle è una piccola ed isolata unità geografica all'interno della Contea di Dubrovnik e Neretva, che si trova nell'estremo sud-est della Croazia la quale confina qui con due paesi limitrofi: Montenegro e Bosnia-Erzegovina: Si estende da nord-ovest a sud-est, per $35 \mathrm{~km}$ in lunghezza, mentre la larghezza massima è di $12 \mathrm{~km}$. Comprende il territorio da Duboka Ljuta a Capo Oštro in Prevlaka (confine con il Montenegro), dalla costa fino al confine con la BosniaErzegovina. Oltre alla terraferma (che comprende Konavoska brda, Gornja e Donja banda) a Konavle appartengono anche cinque piccole isole disabitate..$^{5}$ La posizione geografica della zona relativamente piccola $\left(209,15 \mathrm{~km}^{2}\right)$ e la sua specifica forma di amfiteatro sottolineano l'unicità e la distintività di Konavle nel suo complesso. Anche se il popolamento della zona è continuo fin dalla preistoria, il processo della creazione della specifica identità locale di Konavle fu probabilmente iniziata (nel vero senso), dalla fusione di Konavle alla Repubblica di Ragusa nella prima metà del XV secolo. Secondo l'ultimo censimento della Repubblica della Croazia del 2011, in Konavle vivono 8.577 abitanti (99,35\% cittadini croati) $(\mathrm{DSZ}, 2013)^{6}$. Siccome si tratta della zona prevalentemente rurale, la maggior parte dei residenti abita nei 33 villaggi, dei quali il più grande è il villaggio di Čilipi (934) e solo una piccola parte della popolazione di Konavle abita a Cavtat (2143), unica città di questa microregione (DSZ, 2011).

Fig. 1: La mappa di Konavle (con le sue parti) nel XX secolo ${ }^{7}$

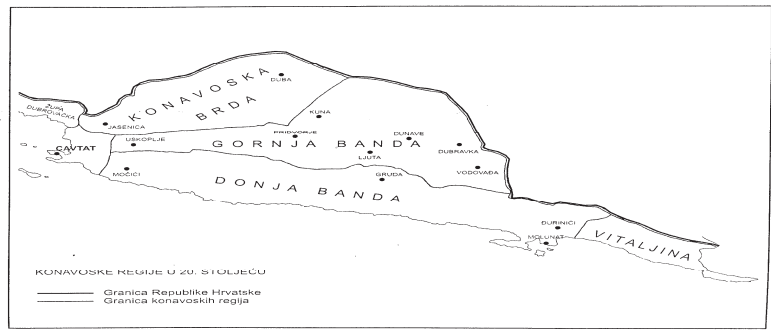

2 La ricerca è stata condotta con lo scopo di preparare la tesi di dottorato: Ravvivazione delle tradizioni: studio antropologico culturale sul turismo culturale in Konavle - Università di Zagabria, Croazia.

${ }^{3}$ Tre inchieste in croato ed una in inglese.

${ }^{4}$ Dati personali su età, sesso, professione, e per i turisti su nazionalità.

${ }^{5}$ Supetar, Mrkan, Bobara, Mali škoj e Veliki škoj.

${ }^{6}$ Ufficio centrale di statistica della Repubblica di Croazia.

${ }^{7}$ Carta $n^{\circ} 6$ - Le parti di Konavle nel XX secolo (Kapetanić \& Vekarić, 1999, p. 13) 
La specifica identità locale limitata alla zona di Konavle si acquista ancor oggi, (secondo la percezione della comunità) sopprattutto con la nascita e con il crescere in quella zona (se non sulla base di origine della famiglia). La creazione di questa identità è influenzata da vari elementi soggettivi e oggettivi. Si può dire che l'identità locale-nativa di Konavle implica alcune altre identità, ad esempio l'identità religiosa cattolica romana (95,95\% della popolazione secondo il censimento del 2011) e l'identità nazionale croata (97,09\% croati) (DZS, 2013). II senso d'appartenenza è associato al territorio di Konavle e alla condivisione di un comune destino storico che ha influenzato in modo specifico vita, cutura e costumi tradizionali di questa regione. La religione cattolica è sempre stata sentita in Konavle come una differenza dai vicini ortodossi e musulmani. La posizione di Konavle è caratterizzata quindi dall',adiacenza”, nel senso spaziale e culturale. La vicinanza del mare e l'impatto di Dubrovnik e del Mediterraneo da un lato e dall'altro i monti di Erzegovina, i Balcani e l'impatto delle "tradizioni patriarcali dinarici” (Bošković Stulli, 1982, p. 131), così come molti altri elementi culturali, portati da varie correnti, hanno segnato la cultura tradizionale di Konavle e la sua identità.

\section{Tradizione e Turismo}

La tradizione come la cultura in generale, è complessa in tutti gli aspetti e comprende sia l'aspetto materiale che immateriale. Si tratta di prassi, rappresentazioni, espressioni, conoscenze, competenze, ma riguarda anche strumenti, oggetti, manufatti e spazi culturali associati con le comunità e gli individui, che li riconoscono come parte del loro patrimonio culturale (UNESCO, 2003). Nelle numerose e differenti definizioni della tradizione è presente la concezione della tradizione come il retaggio e il collegamento degli antenati con le generazioni future (Unesco, 2003; Jelinčić, 2000; Oxford dictionaries, 2013; et al.). La tradizione, come una sorta di costante culturale, è basata sul processo del tramandare, cioè sulla trasmissione della cultura in generale, o dei suoi singoli elementi, da una generazione ad altra.

A volte viene identificata con la parte più stretta della cultura, con la cultura spirituale - con costumi, credenze, letteratura popolare, musica, danza ed espressione visiva. In questo senso, il concetto di tradizione viene sostituito dal concetto di folclore" (Jelinčić, 2000, p. 17). Secondo Ben-Amos, la tradizione come la parte della cultura (folclore) o come l'intera cultura "viene regolarmente associata alle precedenti forme di cultura 0 con quelle forme che stanno scomparendo e si atrribuiscono ad essa le caratteristiche di stabilità e volatilità bassa (cioè alla relativa immutabilità)" (secondo Jelinčić, 2000, p. 17). Nonostante il suo ruolo di „portatore di valori storici del passato” (Jelinčić, 2000, p. 22), la tradizione è "costantemente ricreata" (UNESCO, 2003) e si cambia in risposta della comunità alla natura che la circonda, ma anche alle condizioni sociali e storiche. Questo ci indirizza al dinamismo del termine. Così, la tradizione è „la costruzione contemporanea proiettata nel passato per spiegare il presente" (Čapo Žmegač, 1998, p. 18) e rappresenta (in un certo modo) un passato ereditato ideale identificando e riconoscendo le differenze che sono caratteristiche delle persone e dei luoghi.

Ogni comunità usa il proprio passato per trovare in esso la giustificazione della sua esistenza. Dall'insieme del patrimonio storico-culturale sceglie quegli elementi che nel momento preciso corrispondono al suo sistema dei valori ed interessi speciali. A volte si lascia "nel creare delle nuove tradizioni che sarà difficile distinguere dalle tradizioni reinterpretate, cioè dal creare una nuova coscienza della vecchia cultura" (Hobsbawm, secondo Čapo Žmegač, 1998, p. 17). Tuttavia, ,....il potere e la capacità di adattarsi delle vere tradizioni non è da confondere con le ,tradizioni inventate'. Là dove le antiche tradizioni vivono, non c'è bisogno di risvegliarle e inventarle." (Hobsbawm, 2006, p. 144). In caso di Konavle si tratta principalmente dell'adattamento delle antiche tradizioni al nuovo tempo. Ci sono molti elementi della cultura tradizionale che possono essere utilizzate turisticamente. "Caratteristiche etnografiche della cultura materiale (inventario di casa, strumenti, modi di produzione, artigianato), arti del folclore (musica, danza, arti visive, teatro, letteratura popolare), le specifiche d'abbigliamento tradizionale, specialità gastronomiche popolari (cibo e bevande) e costumi" (Rihtman-Auguštin, 1970, p. 4). In questo lavoro, l'attenzione particolare è rivolta a folclore, sericoltura e molitura, a causa della loro grande importanza sia per l'identità che per il turismo culturale di Konavle.

Fig. 2: Folclore a Čilipi Fig. $n^{\circ} 3$ - Presentazione della sericultura Fig. $n^{\circ} 4$ - Mulino Đivanović
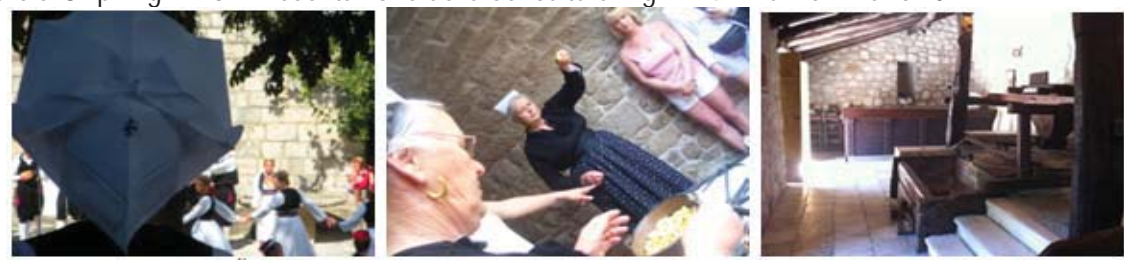

(Z.R.T. 29/8/2000) (Z.R.T. 13/5/2005) (Z.R.T. 8/8/2011) 


\section{Posizione della Tradizione nel Turismo di Konavle}

La tradizione, il tema principale di questo lavoro è menzionata 480 volte nelle risposte degli intervistati nella ricerca del 2010. Più spesso è stata menzionata dai residenti (173 volte), seguito dai turisti (159), guide (90) e il meno dagli operatori turistici (58). La tradizione è raramente menzionata esplicitamente (94), più spesso è usato l'aggettivo tradizionale (e simile) (385) con un sostantivo... Più frequentemente è stata menzionata la cultura tradizionale (231), il modo tradizionale di vita (93) ed i costumi tradizionali (45), ma gli intervistati (16 volte) citano qualcosa altro come tradizionale: principalmente gastronomia $(4)^{8}$, folcore $(3)^{9}$, poi case (2), agriturismi, villaggio, mulino, gioielli, „impegnarsi in un mestiere tradizionale" (A), il comportamento tradizionale e il tradizionalismo in generale.

Oltre a „Tutte le nostre belle tradizioni” (A) sono spesso stati menzionati i costumi (385 volte): vecchi costumi e "Costumi di vecchio stile" (A), tradizioni popolari, precisamente il Carnevale (e la sua variante estiva), costumi matrimoniali e religiosi cioè i costumi tradizionali di Konavle. La maggior parte di essi hanno menzionato i residenti (301), seguiti da turisti (53), operatori turistici (25), e di meno dalle guide (6).

Rispetto a molte semplici menzioni di tradizione (e simile), ci sono di meno le risposte più dettagliate $(40)^{10}$ date nel 2010 dagli intervistati anonimi $(A)$ ed elencate di seguito. La tradizione è più comunemente menzionata in un contesto positivo, mentre le critiche sono pochissime $(7)^{11}$.

Gli operatori turistici (3) raramente rivelano di più sulla tradizione e sulla sua percezione della stessa. Parlando della creazione dei programmi di escursioni in Konavle uno degli intervistati mette in evidenza quello che dovrebbe essere una sorta di regola non scritta: „....cerco di far conoscere ai turisti Konavle nel suo complesso, con particolare attenzione ad agriturismi, costumi, tradizioni e folclore, a seconda del loro tempo libero e budget" (A). Un altro operatore turistico precisa quello che è della migliore qualità del turismo in Konavle: "La parte che coinvolge di più tradizioni (Đivanović, artigianato, Glavić), seta” (A), e il terzo raccomandando il meglio del turismo di Konavle, elenca: „Bisogna vedere i costumi di Konavle e gioielli, mulini, assaggiare la tipica peka e sperimentare l'attuale e precedente modo di vita tradizionale del popolo di questa regione" (A).

Le guide nelle loro risposte più specifiche (10), menzionano due volte esplicitamente la storia di tradizioni onnipresente nel campo. Prevalentemente si tratta di una combinazione, come "Tradizione, modo di vivere, salvaguardia del patrimonio, gastronomia” (A), si risalta „Le escursioni migliori sono quelle in cui i clienti sentono e sperimentano: ...il patrimonio culturale e storico. Preservazione di costumi e tradizioni..." (A) e si specifica „Produzione di seta, il vecchio mulino restaurato e le case in cui si può veramente evocare il modo di vita tradizionale (attrezzi, utensili da cucina...)” (A). Alle guide piacciono: „La varietà dell'offerta e che alcuni mestieri e agriturismi mantengono il tradizionalismo e la specificità di questa zona” (A), „Le visite degli agriturismi dove agli occhi degli stranieri (e di noi) ravvivano antiche tradizioni, artigianato...” (A), „le gite che includono, soprattutto, un po' di tutto' - la visita ad una casa tradizionale, costumi, danze, prodotti fatti in casa" (A). Oltre le menzioni prevalentemente positive della tradizione, le guide hanno dato due menzioni della tradizione in un contesto negativo, criticando l'agriturismo: „Agli ospiti piacciono gli agriturismi, principalmente la casa Glavić, e la casa del pittore Mijo, che hanno ciascuno una personalità per sé stessi, mentre altri agriturismi sono arredati correttamente, ma senza un spirito particolare o un sigillo della tradizione” (A), „Non è utilizzato abbastanza, né sufficientemente ben progettato. L'idea di visitare i complessi residenziali-commerciali è buona ed ottima, ma purtroppo qui manca ,anima' e tradizione, e governa negligenza e disorganizzazione" (A).

Nelle risposte dei residenti si distinguono per unicità le seguenti (20): raccomandando il lavoro nel turismo due intervistati esprimono una visione diversa del turismo: „L'occasione per una presentazione di qualità della propria regione (patrimonio culturale, tradizioni)” (A) e „La possibilità di un approccio diverso alla presentazione del patrimonio culturale e storico” (A). Un residente osserva che i turisti „Apprezzano di più la tradizione e la cultura della gente del luogo e ammirano le risorse naturali di Konavle" (A). Si distinguono per l'importanza e per i dettagli le risposte dei residenti che parlano dei cambiamenti nella cultura tradizionale causati dal turismo. Particolarmente interessanti sono i seguenti pareri contraddittori: una forte posizione conservatrice che rifiuta l'esistenza dei cambiamenti: „Penso che la tradizione e le pratiche religiose siano difficili da cambiare, e penso che questo sia buono" (A) e due critiche negative che testimoniano

\footnotetext{
${ }^{8}$ Gli intervistati hanno menzionato: "La tradizionale cucina di Konavle” (A), raccomandando precisamente "assaggiare la peka tradizionale” (A) e „bevande tradizionali” (A).

9 In particolare: „Ie danze e i costumi tradizionali” (A), „la danza tradizionale Lindo” (A) e „gli spettacoli folcloristici tradizionali”(A).

${ }^{10}$ Le risposte dettagliate sono state fornite da: 20 residenti, 14 guide, 3 operatori turistici e 3 turisti.

${ }^{11}$ Le critiche hanno dato: 2 guide, 2 residenti e 3 turisti.
} 
di loro: „Le persone trascurano spesso la tradizione a causa del lavoro nel turismo, ad esempio le feste dei villaggi e quelle dei santi protettori di famiglia...” (A), „Si tengono i singoli costumi, ma potrebbero essere organizzati un po' meglio, cioè in misura maggiore " (A). Più spesso, però, gli abitanti locali menzionano cambiamenti, e la tradizione in un contesto positivo. II forte legame tra la tradizione e il turismo è esplicitamente espresso tre volte. Si nota che: „... a causa del turismo si introducono di più i vecchi costumi, i mestieri e si aprono gli agriturismi” (A), si cita l'esempio di Čilipi12 menzionato nel contesto del mantenimento della tradizione: „... sempre più persone giovani mantengono i costumi, le persone sono più occupate con il turismo ed espongono i costumi e le antichità" (A). II rapporto tra la tradizione e il turismo svelano le risposte nelle quali non viene menzionato il turismo, ma si sottointende, ad esempio „È tornato a Čilipi il vecchio costume di ballare il folclore la domenica dopo la messa” (A), „Le date non cambiano, i vecchi costumi si svegliano" (a), „Alcuni costumi che si svolgevano prima per le occasioni speciali adesso si presentano ai turisti come l'offerta culturale della zona e sono i cambiamenti positivi” (A). „Dobbiamo rappresentare la nostra identità tramite tradizione, costumi e modo di vita” (A). Un abitante di Konavle nota: „Si fondano diverse associazioni che con i loro programmi cercano di mantenere e là dove è necessario ripristinare la tradizione" $(A)$, un altro cita l'esempio di una manifestazione: „I profumi di Natale' in Konavle diventano una tradizione” (A). Un abitante locale ritiene: „Bisogna comportarsi tradizionalmente anche se si vive velocemente, quindi non è facile armonizzare tutto" (A), altro rileva: "Cercano ${ }^{13}$ di mantenere i vecchi costumi culturali e religiosi" (A), mentre due intervistati precisano i seguenti modi di mantenere i costumi: „Sviluppando nei bambini l'amore per la terra ed i costumi” (A) e "Inserendo le generazioni più giovani nel mantenere costumi, nelle prove quotidiane di danza e coro" (A). Alla fine bisogna sottolineare la conclusione di un abitante di Konavle sul rapporto tra la tradizione e il turismo: „La popolazione di Konavle, semplicemente, non permette che si dimentichino facilmente i costumi, i cambiamenti ci sono ma in gran parte si tramandano dalla generazione alla generazione e si rispettano i costumi" (A).

Tra le risposte degli intervistati turisti, dalle numerevoli semplici menzioni della tradizione (e simile) nel contesto positivo, si distinguono tre critiche. Due volte vengono menzionati gli stessi elementi della cultura tradizionale, criticando: "Il pasto falso e il canto non popolare” (A) e consigliando „È necessario sviluppare il canto autentico e il cibo migliore” (A). Particolarmente interessante è un'osservazione che nota: "L'impatto negativo del turismo sulla popolazione locale che perde il contatto con la cultura tradizionale" (A). Anche se gli intervistati hanno menzionato tradizione molte volte, solo tre guide hanno menzionato i nomi di due concreti meritevoli abitanti di Konavle, notando l'importanza speciale di Antonia Rusković (artista) e Ivo Đivanović (molitore di Ljuta) per mantenere la tradizione e la sua presentazione ad altri.

\section{Folclore nel Contesto Turistico}

II folclore è accordato con la mentalità, la psicologia e lo stile di vita della gente di Konavle che lo impara e l'adotta e poi crea, esegue e trasmette alle generazioni più giovani. Oltre alle abituali funzioni di soddisfare una varietà di bisogni psicologici, sociali e culturali, nel racconto del folclore di Konavle viene sottolineata la sua funzione di rappresentare le tradizioni di Konavle e di „sé stessi” agli altri. La ricerca eseguita ha confermato l'importanza cruciale del folclore per l'identità di Konavle, ma anche per il turismo della stessa regione di Konavle. Due villaggi in Konavle: Čilipi e Pridvorje organizzano gli spettacoli per i turisti. Per il folclore contemporaneo di Konavle in generale (e così anche nel contesto turistico), e specialmente per il folclore di Čilipi di domenica, il ruolo più grande ha la Società Artistico-Culturale „Čilipi” (fondata nel 1954) che ha organizzato la prima rappresentazione folcloristica per i turisti nel 1967. Con questo evento è iniziata non solo "la nuova era per la vita di Čilipi" (Čilipi folklor, 2012), ma anche per la vita del folclore di Konavle. A Čilipi ogni domenica, dalla Dominica delle Palme fino all'ultima domenica di ottobre, si svolge una sorta di trasformazione del villaggio per i turisti, ma anche per i residenti. Si risveglia la tradizione, si organizza lo spettacolo folcloristico e il villaggio si trasforma per una mattina all'attrazione turistica. L'intero programma con il quale si presenta una parte della richezza di Konavle (danze e canzoni locali e un dettaglio dei costumi di nozze), è corto, chiaro, preparato ed eseguito correttamente. Dura all'incirca 45 minuti e finisce abbastanza bruscamente - con la fine del valzer le guide invitano i propri gruppi a tornare in pullman e lasciano il villaggio. II mettere in ordine della scena turistica (secondo l'inglese), setting dura poco e se venite al villaggio la domenica dopo le dodici e trenta indovinerete difficilmente che cosa si svolgeva la stessa mattina sulla piazza davanti alla chiesa di San Nicola. La pace del villaggio semplice di Konavle vince sempre di nuovo l'effimera folla turistica. C'è la simbologia in quello?

\footnotetext{
${ }^{12}$ Citato nel capitolo sul folclore.

13 Pensando ai residenti di Konavle (N.d.A.).
} 
II contrasto tra Čilipi per i turisti e il solito villaggio di Čilipi che si può sperimentare il resto della settimana, come anche lo smontaggio visibile della scena impostata per i turisti, riconfermano gli spettacoli folcloristici di domenica già evidenti davanti ai turisti. Čilipi come il villaggio che si rappresenta ai turisti è diverso e più vivido del villaggio di Čilipi quotidiano, è più di Konavle o è più marcato come di Konavle. Più persone sono vestite nei costumi di Konavle, ${ }^{14}$ si balla, si canta, e si rappresentano tradizioni e costumi del posto, che sicuramente non è (quello che è) quotidiano e solito oggi, nemmeno nella regione stessa di Konavle. Grazie al folclore, Čilipi per i turisti dice loro più dell'identità e della tradizione di Konavle di quanto direbbe qualsiasi altro villaggio (compreso il villaggio di Čilipi stesso) in una situazione ordinaria. Čilipi a questo proposito rientra nella categoria della zona turistica di MacCannell, che si può „chiamare scenografia, impostazioni turistiche o impostazioni, set, a seconda di quanto la visualizzazione stessa è deliberatamente ideata per $\mathrm{i}$ turisti" (MacCannell, 1989, p. 100). Le zone turistiche sono collegate dalle seguenti caratteristiche: l'unica ragione per visitarle è proprio „per vederle”, „fisicamente sono situate vicino alle serie attività sociali oppure le serie attività sociali sono imitate in esse" e di solito contengono, cioè rappresentano ciò che altrimenti non è disponibile agli occhi del pubblico, almeno per un certo tempo, previsto per i visitatori che MacCannell chiama (Ing.) Outsiders (1989, p. 100), gli estranei, intrusi, sottolineando la loro non-appartenenza ai siti che vengono visitati. Si deve sottolineare che spesso le scene, le rappresentazioni turistiche (e simili) "non sono solo copie o repliche delle situazioni della vita reale ma le copie così rappresentate da dire sulla cosa giusta più che la cosa stessa rivela da sola" (MacCannell, 1989, p. 102).

Considerata la natura transitoria che caratterizza il turismo in generale, e in particolare il tempo specifico che i turisti hanno a disposizione (a Čilipi), è necessario riflettere sulla necessità dello stesso tipo, e interrogarsi sull'intelligibilità e sulla giustificazione del risveglio „istante” delle tradizioni locali di Konavle e di un certo „mix per il prezzo del biglietto che si paga" (A). Personalmente non vedo niente di negativo nel ricompenso materiale per la danza e per il canto. II compenso materiale per le prestazioni in etnologia croata non è niente di nuovo... ricordiamoci del dono del corteo per la danza e il canto o la danza con la sposa per raccogliere le risorse materiali per le giovani famiglie (Marošević \& Zebec, 1998, p. 232). Perché ballare per i turisti sarebbe diverso?

II folclore di Konavle a Čilipi parla francamente dell'identità locale e risveglia i suoi sentimenti. Esso vive veramente e non è solo una speciale espressione „istantanea” dell'identità di Konavle, anche se, come tale rappresenta bene la tradizione di Konavle e certamente non è di minore valore questo folclorismo d'oggi che il folclore di una volta, perché le circostanze sono cambiate, ed esso si è adattato al tempo limitato turistico. Parlando dei cambiamenti nella cultura tradizionale la gente di Konavle menziona più spesso il folclore nel contesto positivo che negativo. Tuttavia vanno menzionati anche i pochi cambiamenti negativi nella percezione dei residenti locali: „Gli spettacoli folcloristici si svolgono esclusivamente per i turisti (allontanamento dalla danza originale)...” (A), „Molti cambiamenti, negativi e positivi” (A) accentuando: „Il peggio è che nella rappresentazione della zona si è allontanato dalle vecchie canzoni originali, dai costumi ed in una parola dall'identità (siamo entrati nella società del consumo), l'esempio più eclatante è il folclore in Čilipi che è diventato un mix per il prezzo del biglietto che si paga" (A). Tra le risposte in cui il turismo è identificato come un fattore di cambiamenti positivi spicca il seguente: „Il turismo a Čilipi ha contribuito, attraverso spettacoli folcloristici dal 1967, a preservare la propria identità, i costumi, la produzione tessile, il patrimonio culturale ed altro" (A).

\section{La Sericoltura di Konavle leri, Oggi e Domani}

Il forte senso dell'identità locale di Konavle è ancor oggi più visibilmente espresso con il costume, la cui ricchezza e bellezza sono impensabili senza la seta che in Konavle si produceva nel passato in famiglia e per le sue esigenze. II semplice modo antico della produzione di seta per la tessitura domestica e ricami, si tramandava oralmente attraverso le generazioni, in una sorta di „cornice protetta dalla tradizione” (Gusić, 1970, p. 2). Sotto l'influsso dei grandi e veloci cambiamenti del XX secolo, la sericoltura stava scomparendo lentamente, per essere inaspettatamente rianimata durante gli anni Novanta, ispirata dall'esperienza della guerra (Režić Tolj, 2006), durante la quale una grande parte di costumi e ricami è stata deliberatamente distrutta. Nel 2004 una decina di famiglie si occuppava con la sericoltura ravvivata (Krce, 2004, p. 102). In realtà bisogna sottolineare che sono esclusivamente le donne che si occuppano di seta e sericoltura in Konavle. Le più famose setaiole in Konavle sono due Mare (Mare Drobac e Mare Slovak) a Čilipi che dalla tradizione risvegliata hanno creato una vera e propria attrazione del turismo rurale. Negli ultimi dieci anni, presentavano (a casa Drobac) ai visitatori interessati (per lo più stranieri) una parte del processo della produzione di seta,

${ }_{14}$ Oltre ai danzatori e musicisti, in abiti di Konavle sono vestiti anche altre persone „più di cinquanta attivisti partecipanti al folclore” (Čilipi folklore, 2012). 
come una curiosità che rispetta i valori ambientali di Konavle. Davanti ai turisti si sfilavano i bozzoli e si filavano i fili di seta, cioè si rappresentava quella parte del processo che può essere chiamata "dal bozzolo al filo". L'inizio stesso del processo "dal seme al bozzolo del baco da seta” e altre azioni eseguite con i fili di seta: candeggio, tintura, ricamo e tessitura erano prevalentemente spiegate verbalmente, anche se il ricamo e la tessitura venivano presentati a volte, a seconda dell'interesse del gruppo. Parlando delle loro presentazioni per i turisti le setaiole dicono: „Lo facciamo per tradizione” e spiegano: „La tradizione è forte e rispettata in Konavle. E noi due amiamo la nostra tradizione e la nostra sericoltura, in cui siamo avvolte" (Krce, 2004, p. 103). Anche se il rapporto tra queste donne di Konavli e quest'attività tradizionale rimane forte, gli anni e il tempo portano i cambiamenti... L'immagine idilliaca presentata ha il suo rovescio. L'escursione Via della Seta era il programma raramente eseguito sul repertorio di solo alcune agenzie turistiche locali (Elite, Atlas). Nell'indagine del 2010 la seta e la sericoltura sono state citate 31 volte. Più spesso sono state menzionate dalle guide (17), poi dagli agenti di viaggio (9) e di meno dai residenti (5). Nessuno dei turisti le ha menzionate, probabilmente perché non ha partecipato a questo programma. Tra le risposte spiccano solo due: „L'escursione migliore è stata quella intitolata, ,La storia di seta', in cui in modo diretto e pratico si presentava agli ospiti come si ottiene la seta" (A), mentre l'altra constanta „Mi dispiace solo che per l'escursione ,Via della seta' non ci sia stato mai abbastanza interesse e tuttavia è una delle più originali" (A). Le presentazioni non si tengono a casa Drobac già da due anni, anche se le setaiole ci sperano ancora e la famiglia Slovak allestisce un'area per le presentazioni future.

Attualmente le presentazioni della sericoltura in Konavle sono organizzate soltanto dall'artista locale Antonia Rusković nella sua galleria a Gruda. Con le presentazioni ha iniziato „per caso"15 e le organizza negli ultimi quattro anni, per piccoli gruppi (5-8 persone, anche se possono essere al massimo 35) che portano molte agenzie turistiche di Dubrovnik (Elite, Travel Europe, Aragosa ecc), perlopiù in primavera e in autunno (Antonia Rusković, comunicazione personale, 2013), quando è possibile vedere anche i bachi da seta che rendono le presentazioni più complete e belle. Tuttavia quest'artista è sempre „... pronta a mostrare agli ospiti il baco da seta e a dire qualcosa sui costumi di Konavle e sul suo lavoro nel mantenere viva la tradizione" (A). II suo lavoro porta speranza, favorisce il mantenimento di quest'attività tradizionale tra la gente di Konavle, la rende più popolare tra la gente locale ma anche tra i visitatori stranieri. La sua galleria d'arte è sicuramente il posto migliore per acquistare souvenir di Konavle. II numero, la varietà e la qualità dei prodotti, oltre ad originalità garantita e la produzione in situ, insieme con la possibilità di sentire dalla stessa artista la storia di seta e tradizione di Konavle, rendono questo posto molto speciale tanto da meritare una visita. Sarebbe bene che prendesse la vita l'idea "di valorizzare quel vecchio mestiere come un marchio" (Gović, 2001, p. 44) di (almeno) alcuni agriturismi in Konavle. Così si aiuterebbe a mantenere quest'attività tradizionale generalmente già sparita da altre regioni croate che si inserisce bene in una moderna ricerca turistica per i valori (etno-eco) tradizionali.

\section{Mulini, Frantoi e Gualchiere del Fiume Ljuta nel Contesto Turistico}

La zona del fiume Ljuta era nel passato l'unica area in Konavle dove mulini, frantoi (per le olive) ${ }^{16}$ e gualchiere erano in funzione tutto l'anno. Li usavano salvo la gente di Konavle e gli abitanti della regione circostante (Montenegro, Erzegovina) (Ivo Đivanović, comunicazione personale, 2005). Oggi in Konavle, sul fiume Ljuta esistono tre acquedotti e quindici edifici economici: dodici (case) mulini ${ }^{17}$, tre gualchiere e due frantoi situati all'interno dei mulini. Ci sono anche i resti di altri tre mulini e della quarta gualchiera, mentre dell'esistenza precedente del terzo frantoio, unico a sé stante per le olive (Dobrašin), testimonia solo la corona decorativa davanti alla casa. La maggior parte di questi edifici è stata costruita secondo un piano regolatore, durante la Repubblica di Ragusa (oggi Dubrovnik) ${ }^{18}$, formando un sistema completo. Quando la Repubblica di Ragusa nel 1426 ha acquistato la parte occidentale di Konavle e così finalmente l'intera regione, sul fiume Ljuta sono stati trovati e registrati quattro mulini (Kapetanić, 2012, p. 10). Durante tutta la sua esistenza, la Repubblica costruiva, estendeva, adattava, ma anche abbandonava gli edifici, migliorando semplicemente il sistema dei mulini, gualchiere e frantoi in modo che nel corso dei secoli avesse raggiunto veramente "la massima utilizzazione dell'energia potenziale dell'acqua" (Kapetanić, 2012, p. 45) del fiume Ljuta. A causa di grandi cambiamenti (in stile di vita e lavoro) portati dal XX secolo, si è svolto un progressivo abbandono di quegli edifici economici specialmente nel secondo dopoguerra, che rappresentavano per tanti anni seguenti diverse cattive condizioni della più 0

\footnotetext{
${ }^{15}$ Grazie ai suoi laboratori di pittura e a richiesta delle agenzie turistiche.

${ }^{16}$ I frantoi sono in funzione solo nel tardo autunno, quando le olive si raccolgono e si produce l'olio d'oliva.

17. mulini hanno 22 meccanismi per la produzione di farina.

${ }^{18}$ La Repubblica di Ragusa esistette dal 1358 al 1806 (anche se i francesi l' hanno formalmente abolita nel 1808).
} 
meno grande preservazione e/o degradazione. La percezione del valore di questo patrimonio è migliorato molto negli ultimi 15 anni nei proprietari, ma anche nella gente di Konavle in generale. Tutto è cominciato grazie all'esempio eccellente di Ivo Đivanović che nel 1997 iniziò con le presentazioni turistiche della molitura tradizionale di Konavle. Ho visitato il mulino Đivanović per la prima volta nel 1999 lavorando come accompagnatrice all'escursione chiamata Konavle Valley per un'agenzia di viaggi. Le visite frequenti, negli ultimi anni, aumentavano il mio interesse personale per il particolare argomento, sia in termini di architettura e di scopi commerciali, ma anche in termini del contesto storico, sociale ed economico.

II mulino Đivanović19 è uno dei sei mulini più antichi che la Repubblica di Ragusa ha trovato stabilendo il proprio potere in Konavle (Kapetanić, 2012, p. 25). II mulino ha funzionato normalmente fino agli anni '60 del secolo scorso. Con l'apertura del ristorante Konavoski dvori nel 1968 il mulino (come bar) e la gualchiera della famiglia Đivanović (nelle vicinanze) sono stati inclusi nell'offerta turistica del ristorante (Ivo Đivanović, comunicazione personale, 2005 \& Kapetanić, 2012, p. 56). Secondo l'intervistato Ivo Đivanović gli edifici sono stati precedentemente restaurati e preparati per il turismo sotto la supervisione del Dipartimento di Conservazione a Dubrovnik, mentre i meccanismi molto probabilmente non erano in uso. II mulino è stato ristrutturato dopo la guerra, nel 1997, dal proprietario stesso (famiglia Đivanović) con i mezzi propri e quelli del Ministero del Turismo. Nell'autunno dello stesso anno arrivò il primo gruppo di turisti20. Oggi Ivo lavora con tutte le agenzie turistiche di Dubrovnik, per lo più con Atlas e Elite. Le visite annuali del mulino sono di circa 10.000 persone, mentre il massimo di 15.500 visitatori è stato raggiunto nel 2005 (Ivo Đivanović, comunicazione personale, 2011). Le presentazioni di Ivo in inglese 0 in lingua croata, sono sempre interessanti, brevi e chiare, provocano l'interesse e le domande a cui Ivo, il vero Konavljanin è sempre pronto a rispondere. Le presentazioni del mulino eseguite da Ivo per i turisti e gli altri curiosi, cominciano davanti al mulino. Ivo, indossando l'abbigliamento di Konavle, accoglie gli ospiti e li introduce allo status dell'edificio: „Il mulino è un monumento dal tempo della Repubblica di Ragusa, che esiste dal XVI secolo ed apparteneva al sistema di mulini, frantoi e gualchiere che la Repubblica ha costruito sul fiume Ljuta." (Ivo Đivanović, comunicazione personale, 1997 - ). Invitando i visitatori nel mulino Ivo inizia con la presentazione del suo lavoro, con la quale fa conoscere ai curiosi il mulino come l'edificio, le parti fondamentali del suo meccanismo e i vantaggi di questo modo di produzione della farina. Nel mulino della famiglia Đivanović, accanto all'abituale inventario del mulino, c'è anche il piccolo banco bar che aiuta il finanziamento e la manutenzione del mulino perché l'esistenza di quell'edificio non si può basare né sull'attività primaria del mulino, né solo sulle presentazioni del mestiere tradizionale per il turismo.

Alcuni programmi delle escursioni in Konavle, oltre alla presentazione del mulino Đivanović, includono anche la gualchiera Đivanović situata in un ambiente splendido, su un'isola in mezzo al fiume Ljuta. Grazie agli sforzi del suo proprietario (Niko Đivanović), sull'iniziativa e con l'aiuto dello stesso Ivo Đivanović, l'edificio è stato ripristinato nel 200921, rispettando le norme dell'architettura rurale e della tradizione, ed è stato ricostruito completamente il meccanismo. Oggi la gualchiera si usa soltanto per le presentazioni turistiche ed è inclusa nell'offerta turistica del Mulino. Ivo Đivanović esegue anche le presentazioni della gualchiera ${ }^{22}$ visitata annualmente da allincirca 4.000 - 5.000 persone (Ivo Đivanović, comunicazione personale, 2011.). Gli sforzi enormi e l'approccio straordinario di Ivo Đivanović superano le presentazioni stesse degli edifici economici con le connesse attività tradizionali di Konavle che si svolgono in essi. II miglior complimento allo sforzo e all'approccio di questo Konavljanin insolito è rappresentata dalla risposta di una guida alla domanda su quello che percepisce come migliore offerta turistica in Konavle, che riassume l'esperienza di molti che hanno avuto l'opportunità e la fortuna di incontrare Ivo e il suo lavoro: „Incontrare una persona che apprezza tanto il proprio lavoro, costumi e tradizioni della sua regione, non accade spesso. La conclusione: solo queste persone possono essere meritevoli per una tradizione così ricca e per la conservazione di questi valori a giorno d'oggi. Dunque sono loro i migliori di Konavle così almeno posso concludere dall'esperienza personale" (A).

Oggi, per fortuna, è ricostruito quasi tutto il sistema, ma soltanto il mulino Đivanović è nella reale funzione d'ogni giorno, anche se principalmente nella funzione turistica, unica per la gualchiera Đivanović. II mulino Cucalo (Kapetanić) restaurato nel 2004, opera di tanto in tanto, mentre sta per aprire il mulino Pišta con un frantoio all'interno in fase di lavoro (di conservazione e restaurazione) Altri edifici sono in realtà abbandonati, ma grazie a cura, sforzi e capacità della DPDS - Società degli amici delle antichità di Dubrovnik, non sono più del tutto trascurati. Dal 2005 al 2011 sono stati

\footnotetext{
19 II mulino ha due meccanismi per la produzione di farina.

20 Generalturist ha portato i primi visitatori della agenzia britannica Saga.

${ }^{21}$ La ricostruzione è stata finanziata con i fondi privati e quelli del Ministero della Cultura e del Turismo croato.

${ }^{22}$ Le presentazioni nella gualchiera sono meno frequenti di quelle nel mulino.
} 
rinnovati sette mulini costruiti durante il periodo della Repubblica, i resti di altri due mulini sono stati conservati $($ Kapetanić, 2012, p. 75)23. Infatti, sono stati „restaurati muri e tetti (riparazione strutturale)” degli ediffici, l'interno è stato pulito e conservato, mentre i meccanismi non sono stati rinnovati perché la ricostruzione è guidata „supponendo che sarebbero rinnovati dai proprietari stessi" (Kapetanić, 2012, p. 75). Durante la ristrutturazione si è pensato anche all'ambiente, cercando (quanto possibile) di ottenere la presunta immagine ideale del sistema dei mulini, frantoi e gualchiere del fiume Ljuta nel passato. Camminando per la Via dei mulini, è veramente piacevole vedere gli edifici restaurati dall'esterno (nonostante la situazione meno bella nei loro interni), la sistemazione dell'area circostante e la bellezza che li circonda. Bisogna sottolineare l'importanza della reale (e non solo nominale) ${ }^{24}$ protezione dell'intera zona del fiume, come un insieme naturale e culturale. La conservazione e/o il restauro dell'architettura di questi edifici economici è lodevole, ma non basta per sé. Per farli esistere veramente il fiume Ljuta deve rimanere ecologicamente indisturbata e mulini, frantoi e gualchiere devono essere necessari. Questo si può realizzare soltanto con un agire pensato, comune, sia a livello locale, regionale che nazionale. Ljuta, un'area riconoscibile, un'attrazione locale, come un'eco-etno oasi potrebbe migliorare la vita della zona, creando nuovi posti di lavoro e mantendo le occupazioni tradizionali dei mugnai, frantoiani, addetti alle gualchiere ed agricoltori. Nelle parti trascurate della valle fertile di Konavle, potrebbero di nuovo dorarsi i cereali ottenuti con la coltivazione e trattamento biologico, creando i prodotti agricoli sani, così ricercati nel mondo. I visitatori potrebbero godere della natura incontaminata, immergersi nel mondo idilliaco di rapporto armonico tra l'uomo e il mondo che li circonda e imparare qualcosa sul passato, ma anche da esso.

\section{Su Tradizione, Turismo e Konavle - Di Nuovo}

"L'incontro con le persone reali, con i loro desideri e bisogni, con i loro valori, crisi e conflitti e infine, con la loro creatività è l'unico test valido della teoria." (Rihtman-Auguštin, 1976, p. 21). II lavoro presenta e documenta lo stato attuale, il presente della tradizione di Konavle come un esempio particolare della simbiosi della tradizione e contemporaneità, con $\mathrm{i}$ vivi elementi rappresentativi della specifica locale cultura tradizionale. Si presenta la percezione della tradizione di Konavle nel contesto turistico e si dà l'immagine della fruizione turistica del folclore come elemento selezionato della cultura tradizionale di Konavle e dei fenomeni specifici della ravvivazione delle attività tradizionali (sericoltura e molitura) a scopo del turismo. Esistono anche altri elementi della cultura tradizionale di cui si potrebbe dire molto: gastronomia, architettura rurale ecc. La ricerca e il lavoro sul tema confermano che gli elementi selezionati della cultura tradizionale sono importanti elementi dell'identità culturale e locale della regione di Konavle. Dimostrano anche che le forme culturali e le nozioni di identità della comunità locale persistono e si trasformano (anche) influenzati dalle esigenze turistiche e dalle interazioni tra i partecipanti diversi al turismo. L'esistente offerta turistica rappresenta Konavle come una destinazione turistica con l'accento sulla specifica cultura tradizionale della micro-regione con una forte identità locale. I turisti interessati vengono in questa oasi della vita tradizionale appunto per la diversità che offre. II patrimonio tradizionale, anche se oggi piuttosto confezionato in contenitori di folclore, è una base importante per lo sviluppo di questa regione. II turismo, assicurando l'esistenza di un certo numero di famiglie in Konavle, ha un effetto positivo sul mantenimento dell'identità locale e della cultura tradizionale, nonostante le raffiche sempre più forti della globalizzazione, attraverso varie forme di comunicazione contemporanea.

Non è difficile riconoscere i valori che abbiamo (o abbiamo avuto), ma dobbiamo volere e sapere preservarli e, se necessario, dargli una nuova vita adattandoli alle esigenze moderne, anche quelle turistiche, senza dimenticare però il dovuto rispetto per il loro patrimonio. È necessario quindi, sviluppare una consapevolezza dei valori che esistono grazie all'eredità, ma anche quella dell'obbligo per esso. La ricerca della tradizione di Konavle nel contesto del turismo conferma la sua vitalità, la dinamica culturale e la variabilità in conformità con le esigenze e le richieste della vita moderna. Utilizzando l'esempio della cultura tradizionale di Konavle sono state esaminate alcune delle possibili applicazioni del sapere antropologico culturale / etnologico nel mondo del turismo. Le conoscenze etno-antropologiche possono aiutare il turismo in modi diversi, sia che si tratti semplicemente di una migliore comprensione tra le persone di culture diverse o di qualcosa più complesso ad es. comprensione dellinterazione tra turismo culturale e processi culturali

\footnotetext{
${ }^{23}$ DPDS intende continuare con la ristrutturazione dei restanti 5 edifici (3 mulini e 2 gualchiere).

${ }^{24}$ I mulini del fiume Ljuta sono stati protetti nel 1969 come monumenti culturali, nel 1975 tutta la zona del fiume è stata iscritta nel registro degli oggetti naturali protetti (Kapetanić, 2012). In Croazia il sistema dei mulini, frantoi e gualchiere sul fiume Ljuta era sotto la tutela cautelare, secondo l'ordine del Ministero della Cultura Dipartimento di Beni Culturali Dipartimento di Conservazione a Dubrovnik. La decisione sulla protezione preventiva è scaduta nel 2006, l'emissione di una decisione di protezione permanente di questo bene culturale è in corso (Evidencija..., 2004 \& 2012).
} 
(dell'identificazione) in Konavle. Ma quello particolarmente importante dal punto di vista locale è che il sapere etnologico può aiutare a generare il reddito maggiore di Konavle. Una maggiore applicazione delle conoscenze etnologiche e antropologico culturali della cultura tradizionale Konavle porterebbe a una superiore qualità del turismo culturale in Konavle e ad una migliore rappresentazione dei singoli elementi della cultura tradizionale come specifiche attrazioni turistiche. Nel tipo esperienziale del turismo è importante l'incontro con le risorse naturali e conoscere la cultura, la storia e l',anima” della destinazione. II turismo con il sigillo locale di Konavle promuove (in senso ideale) il valore della responsabilità umana per il patrimonio naturale, culturale e spirituale, rafforza la consapevolezza ambientale, promuove lo sviluppo sostenibile e semplicemente contribuisce al benessere degli esseri umani e dell'ambiente. I programmi che si dovrebbero svolgere in Konavle devono essere indirizzati a creare contenuti con l'origine nella cultura locale, dando precedenza ai valori di cultura tradizionale di Konavle, di artigianato ed arte locale con sempre consigliabile collegamento dei valori ed idee locali con quegli universali.

\section{Literatura}

Bošković-Stulli, M. (1982). Konavosko usmeno pjesništvo. Konavoski zbornik, Dubrovnik 1, 130-139.

Čapo Žmegač, J. (1998). Elementi hrvatske seljačke kulture u prostoru i vremenu. In Čapo Žmegač, J., Muraj, A., Vitez, Z., Grbić, J., Belaj, V., et al. (Eds.), Etnografija: svagdan i blagdan hrvatskoga puka. Zagreb: Matica hrvatska. 9 - 23.

Čilipi folklor (2012). «http://www.cilipifolklor.hr/hrvatski.htm> Consultato il 15 marzo 2012.

DZS (2011). Državni zavod za statistiku Republike Hrvatske. Statistička izvješća 1441/2011. «http://www.dzs.hr/Hrv_eng /publication/2011/SI-1441.pdf). Consultato il 14 dicembre 2011. DZS (2013). Državni zavod za statistiku Republike Hrvatske.shttp://www.dzs.hr> Consultato il 30 maggio 2013.

Evidencija iz dokumentacije kulturnih dobara za područje Konavala (2004 \& 2012). Ministarstvo kulture, Uprava za zaštitu kulturne baštine, Konzervatorski odjel u Dubrovniku.

Goffman, E. (2000). Kako se predstavljamo u svakodnevnom životu. Beograd: Geopoetika.

Gović, T. (2001). Konavle - koljevka svilarstva. Hrvatski iseljenički zbornik, 40 - 46.

Gušić, M. (1970). Svila u vezu i tkanju na dalmatinskom narodnom ruhu, izložba IX, Etnografski muzej u Splitu (s.p.).

Hobsbawm, E. (2006). Izmišljanje tradicije. In Brkljačić, M. \& Prlenda, S. (Eds.), Kultura, pamćenje i historija. Dialogica Europea. Zagreb: Golden marketing. - Tehnička knjiga. $138-150$.

Jelinčić, D. A. (2000). Kulturna baština i turizam. Magistarski rad. Sveučilište u Zagrebu. Filozofski fakultet. Zagreb. IEF rkp. 1715.

Kapetanić, N. i Vekarić, N. (1999). Stanovništvo Konavala 2. Stipetić, V. (Ed.), Dubrovnik: Zavod za povijesne znanosti HAZU u Dubrovniku.

Kapetanić, N. (2012). Mlinovi na rijeci Ljutoj u Konavlima. Dubrovnik: Društvo prijatelja dubrovačke starine i Gruda: Matica hrvatska, Ogranak Konavle.

Krce, S. (2004). Konavoski put svile. National geografic Hrvatska (dicembre), 99 - 104.

MacCannell, D. (1989). The tourist. A New Theory of the Leisure Class. New York. Schocken books.

Marošević, G. \& Zebec, T. (1998). Folklorna glazba i ples. In Čapo Žmegač, J., Muraj, A., Vitez, Z., Grbić, J., Belaj, V., et al. (Eds.), Etnografija: svagdan i blagdan hrvatskoga puka. Zagreb: Matica hrvatska. 231, 232.

Oxford dictionaries (2013). «http://oxforddictionaries.com/definition/english/tradition> Consultato il 15 maggio 2013.

Režić Tolj, Z. (2007). Svilarstvo u Konavlima, oživljavanje jedne tradicije. Etnološka tribina, nº 30, 95 - 117.

Rihtman-Auguštin, D. (1970). Tradicionalna kultura i turizam. Dometi 6, 4 - 11.

Rihtman-Auguštin, D. (1976). Pretpostavke suvremenog etnološkog istraživanja. Narodna umjetnost, nº 13, 1 - 23.

Simonicca, A. (2001). Antropologia del turismo. Roma: Carocci editore S.p.A.

Unesco (2003). «http://www.unesco.it/cni/index.php/cultura/patrimonio-immateriales Consultato il 29 aprile 2013. 